\title{
Investigating the influence of continuous babble on auditory short-term memory performance
}

\section{Document Version}

Final published version

Link to publication record in Manchester Research Explorer

\section{Citation for published version (APA):}

Heinrich, A., Schneider, B. A., \& Craik, F. I. M. (2008). Investigating the influence of continuous babble on auditory short-term memory performance. The Quarterly journal of experimental psychology, 61(5), 735-751.

\section{Published in:}

The Quarterly journal of experimental psychology

\section{Citing this paper}

Please note that where the full-text provided on Manchester Research Explorer is the Author Accepted Manuscript or Proof version this may differ from the final Published version. If citing, it is advised that you check and use the publisher's definitive version.

\section{General rights}

Copyright and moral rights for the publications made accessible in the Research Explorer are retained by the authors and/or other copyright owners and it is a condition of accessing publications that users recognise and abide by the legal requirements associated with these rights.

\section{Takedown policy}

If you believe that this document breaches copyright please refer to the University of Manchester's Takedown Procedures [http://man.ac.uk/04Y6Bo] or contact uml.scholarlycommunications@manchester.ac.uk providing relevant details, so we can investigate your claim.

\section{OPEN ACCESS}


This article was downloaded by: [University of Nottingham]

On: 26 January 2011

Access details: Access Details: [subscription number 789284405]

Publisher Psychology Press

Informa Ltd Registered in England and Wales Registered Number: 1072954 Registered office: Mortimer House, 3741 Mortimer Street, London W1T 3JH, UK

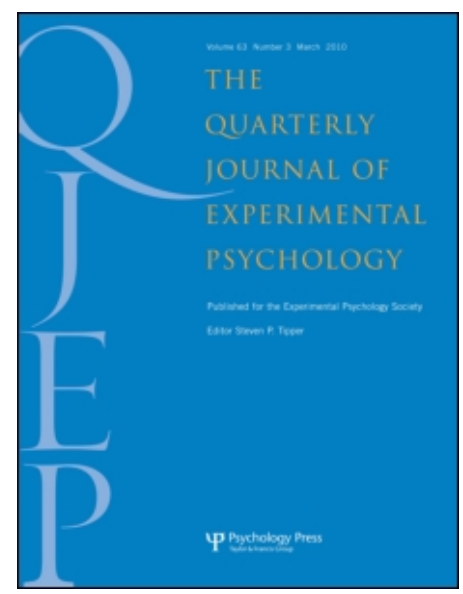

\section{The Quarterly Journal of Experimental Psychology}

Publication details, including instructions for authors and subscription information:

http://prod.informaworld.com/smpp/title $\sim$ content=t716100704

\section{Investigating the influence of continuous babble on auditory short-term memory performance}

Antje Heinrich ${ }^{\mathrm{a}}$; Bruce A. Schneider ${ }^{\mathrm{a}}$ Fergus I. M. Craik

${ }^{\mathrm{a}}$ University of Toronto at Mississauga, Mississauga, Ontario, Canada ${ }^{\mathrm{b}}$ Rotman Research Institute, Toronto, Ontario, Canada

First published on: 27 July 2007

To cite this Article Heinrich, Antje, Schneider, Bruce A. and Craik, Fergus I. M.(2008) 'Investigating the influence of continuous babble on auditory short-term memory performance', The Quarterly Journal of Experimental Psychology, 61: 5, 735 - 751, First published on: 27 July 2007 (iFirst)

To link to this Article: DOI: $10.1080 / 17470210701402372$

URL: http://dx.doi.org/10.1080/17470210701402372

\section{PLEASE SCROLL DOWN FOR ARTICLE}

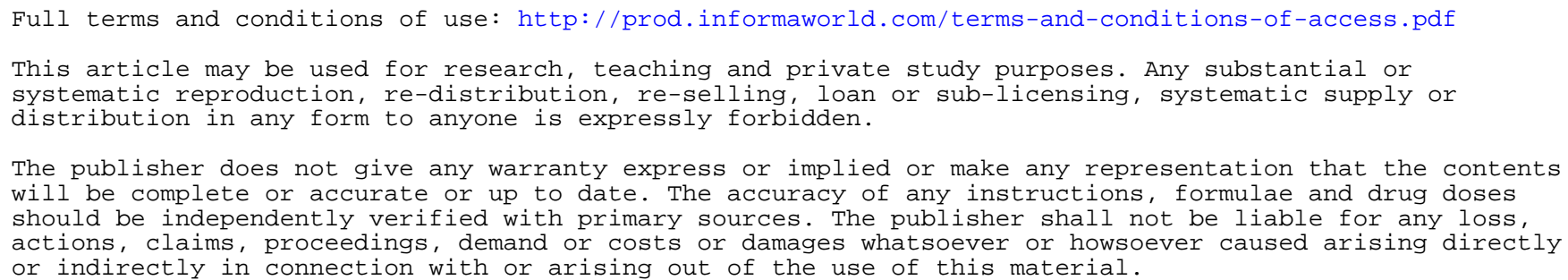




\title{
Investigating the influence of continuous babble on auditory short-term memory performance
}

\author{
Antje Heinrich and Bruce A. Schneider \\ University of Toronto at Mississanga, Mississanga, Ontario, Canada \\ Fergus I. M. Craik \\ Rotman Research Institute, Toronto, Ontario, Canada
}

\begin{abstract}
A number of factors could explain the adverse effect that babble noise has on memory for spoken words (Murphy, Craik, Li, \& Schneider, 2000). Babble could degrade the perceptual representation of words to such an extent that it compromises their subsequent processing, or the presence of speech noise in the period between word presentations could interfere with rehearsal. Thirdly, the top-down processes needed to extract the words from the babble could draw on resources that otherwise would be used for encoding. We tested all these hypotheses by presenting babble either only during word presentation or rehearsal, or by gating the babble on and off $500 \mathrm{~ms}$ before and after each word pair. Only the last condition led to a decline in memory. We propose that this decline in memory occurred because participants were focusing their attention on the auditory stream (to enable them to better segregate the words from the noise background) rather than on remembering the words they had heard. To further support our claim we show that a similar memory deficit results when participants perform the same memory task in quiet together with a nonauditory attention-demanding secondary task.
\end{abstract}

\section{Memory deficits in auditory tasks - the case for auditory complexity}

The degree to which we can successfully comprehend and remember auditory information depends on a number of factors. For example, we know that our ability to remember heard information will depend on the complexity of the material, the speed at which it is presented, the attention devoted to its processing, and, in some instances, the age of the listener. In addition, it is also the case that the complexity of the auditory environment in which the to-be-remembered information is presented can adversely affect comprehension and memory of heard material. Whereas a large number of studies have examined the former factors, there have been relatively few laboratory studies of how noise in the auditory

Correspondence should be addressed to Antje Heinrich, MRC Cognition and Brain Sciences Unit, 15 Chaucer Road, Cambridge, CB2 7EF, UK. E-mail: antje.heinrich@mrc-cbu.cam.ac.uk

The research in this article was supported by grants from the Natural Sciences and Engineering Research Council of Canada and Canadian Institutes of Health Research. The first author was supported, in part, by a Canadian Institutes of Health research training grant, and by the International Council for Canadian Studies. We thank Jane Carey and Neda and Alham Chelehmalzadeh for their assistance in data collection. 
environment affects memory, in spite of the ubiquity of noise in everyday situations. Those studies that have appeared in the literature, however, do indicate that background noise interferes with memory of unrelated items, sentences, or discourse, and that such interference can be observed when the acoustic background consists of random noise, other talkers, or babble (many different people talking simultaneously) (Murphy, Craik, Li, \& Schneider, 2000; Murphy, Daneman, \& Schneider, 2006; Peelle \& Wingfield, 2005; Pichora-Fuller, Schneider, \& Daneman, 1995; Rabbitt, 1968, 1991; Schneider, Daneman, Murphy, \& Kwong-See, 2000). The reasons why memory is affected by noise are less clear, however.

\section{Energetic masking versus top-down influences}

One possibility is that noise simply masks the tobe-remembered material. After all, it is difficult to recall an item or piece of information that is "drowned out" or masked by the noise because the noise activates the same regions on the basilar membrane as does the speech target. Such masking is often referred to as "energetic" masking because the energy in the noise masks the energy in the target (see Brungart, 2001, for a review).

However, a number of results suggest that energetic masking cannot be the only reason for the memory deficit. For instance, Rabbitt (1968) showed that memory for spoken digits in normalhearing participants was adversely affected by the presence of noise, even when the level of noise permitted near-perfect recognition of the individual digits. Even more strikingly, he observed a similar drop in memory when the items following the targets (but not the targets themselves) were perceived through background noise. Similarly, Murphy et al. (2000), using a paired-associate memory paradigm, found that while intelligibility of the words presented in a 12-talker babble dropped by only $9 \%$ compared to quiet, memory performance dropped by up to $50 \%$. Lastly, a few studies looking at hearing loss and stimulus distortion demonstrate that memory deficits can occur in the absence of perceptual deficits, supporting the idea that the effect of an adverse listening situation may not become apparent until later stages of processing. For instance, Rabbitt (1991) showed that individuals with good hearing recalled more words than did individuals with mild hearing loss, even when both groups were equally accurate in repeating the words that they heard. If peripheral masking was solely responsible for memory loss in noise, then we would expect that when two groups were matched with respect to the accuracy with which they could repeat words, they would perform equivalently on the memory task. Similarly, Luce, Feustel, and Pisoni (1983), using natural and synthetic speech, showed that synthetic speech led to a memory deficit but not to a perceptual deficit.

If energetic masking cannot explain these memory effects, we must look to other processes for an explanation. One hypothesis is that the adverse effects that babble (Murphy et al., 2000), hearing loss (Rabbitt, 1991), and stimulus distortion (Luce et al., 1983) have on memory is a consequence of perceptual degradation. According to this hypothesis, hearing loss, background babble, and synthetic speech all degrade the sensory registration of the lexical items to such an extent that they interfere with the encoding of the items in memory. McCoy et al. (McCoy, Tun, Cox, Colangelo, Stewart, \& Wingfield, 2005, p. 22) refer to this as the "effortfulness hypothesis": the notion that the extra effort that a hearing-impaired listener must expend to achieve perceptual success comes at the cost of processing resources that might otherwise be available for encoding the speech content in memory.

Another hypothesis as to how noise might affect memory is that involuntary processing of the information in the masker may interfere with the cognitive processes involved in the encoding and rehearsal of to-be-remembered items. In the psychoacoustic literature, cognitive level interference of this sort is often referred to as informational masking (Durlach, Mason, ShinnCunningham, Arbogast, Colburn, \& Kidd, 2003; Freyman, Balakrishnan, \& Helfer, 2001; Li, 
Daneman, Qi, \& Schneider, 2004). For example, when the background noise is 12-talker babble, the babble itself may elicit phonological and semantic processing that interferes with the encoding and rehearsal of words. If such phonological and semantic processing of the noise background cannot be inhibited (e.g., Hasher \& Zacks, 1979; Hasher, Zacks, \& May, 1999), listeners would, in fact, be performing a secondary task while trying to rehearse or remember the heard words.

A third possibility as to why background babble affects memory is based on the concept of stream segregation and assumes that the presence of noise necessitates the segregation of target items and babble into different perceptual streams (Bregman, 1990). It is possible that participants, while attempting to memorize items in babble, might also have to continuously segregate the two streams to optimize the extraction of the word pairs from the background. As several recent studies have shown, the degree to which streaming occurs can be affected by attention (Alain \& Izenberg, 2003; Carlyon, Plack, Fantini, \& Cusack, 2003; Sussman, Winkler, Huotilainen, Ritter, \& Näätänen, 2002). If the segregation of different sound sources into separate perceptual streams is attention-demanding, it is possible that the redeployment of limited attentional and other processing resources towards the extraction of words from a noisy auditory scene could interrupt rehearsal by depleting the pool of resources available to higher-level, more cognitive stages of processing, thereby resulting in lower recall. This last interpretation could provide a basis to explain another detail of studies in which the material to be recalled was presented serially-namely that the effect of noise and stimulus distortion on memory performance was much more pronounced for those items presented earlier rather than later. For example, looking at the group of young adults tested by Murphy et al. (2000), babble affected memory only in the early serial positions in the paired-associate memory paradigm. In fact, when tested at different signal-to-noise ratios (SNRs), memory performance did not decrease equally for all positions on the list; instead, the first three serial positions were more strongly affected than the fourth and fifth positions. In the present paper, we argue that the diversion of attentional resources to facilitate auditory streaming in a listening environment with background noise will lead to a shallower encoding of words, which will primarily affect the words presented earlier in the list.

\section{Augmenting the comparison groups}

In the present study, the effects of the new manipulations were contrasted with baseline data collected under conditions of quiet and continuous babble obtained from the experiment reported by Murphy et al. (2000). We had also conducted a further experiment under these identical conditions, however, and since this further study replicated the original experimental results, we combined the data to provide a more stable basis of comparison. The further study involved participants for whom English was a second language (ESL). Previous studies have reported that cognitive processes are slower and more effortful in the second language (Ardila, 2003; Ardila et al., 2000), so we wished to explore whether ESL participants who were totally fluent in English might nevertheless show memory problems on the paradigm documented by Murphy et al. (2000). Our ESL group all spoke English fluently; they acquired the language between the ages of 7 and 13 years, at which point they moved to an English-speaking country and did all their schooling in English.

The fact that our ESL group had acquired English relatively late in life manifested itself in two measures: compared to their fellow students who had learned English as a first language before age 5 , second-language speakers had a reduced Mill Hill vocabulary score $(M=11.55$, $S E=0.47$ compared to $M=13.35, S E=0.61$ for first-language speakers) and required a higher SNR to reach a threshold criterion ( $50 \%$ correct) for identifying the final word in a set of sentences $(M=2.61, S E=0.52$ compared to $M=-0.13$, $S E=0.52)$. In this replication, 15 secondlanguage participants were tested in the Murphy et al. (2000) paradigm (modelled after Madigan 
\& McCabe, 1971) in a quiet background, and another 18 second-language participants were tested in a background of continuous babble. The equipment, material, and testing procedures (which involved individual adjustment of the SNR to account for differences in word-recognition thresholds) were identical to those in the original study (see the Method section below for a more detailed description). The results from this replication (see Figure 1) indicated that memory performance in both quiet and babble is independent of the language status of the participants. A mixed-measures analysis of variance (ANOVA) with serial position as a withinsubject factor and noise condition (quiet vs. babble) and language status (first vs. second language) as between-subject factors, found significant main effects for serial position, $F(4,236)$ $=91.01, M S E=284.83, p<.0001$, and noise condition, $F(1,59)=10.87, M S E=13,197.84$, $p=.002$, as well as a significant interaction between the two factors, $F(4,236)=3.78, M S E$ $=284.83, p=.005$. However, the age at which English was acquired had no effect on memory

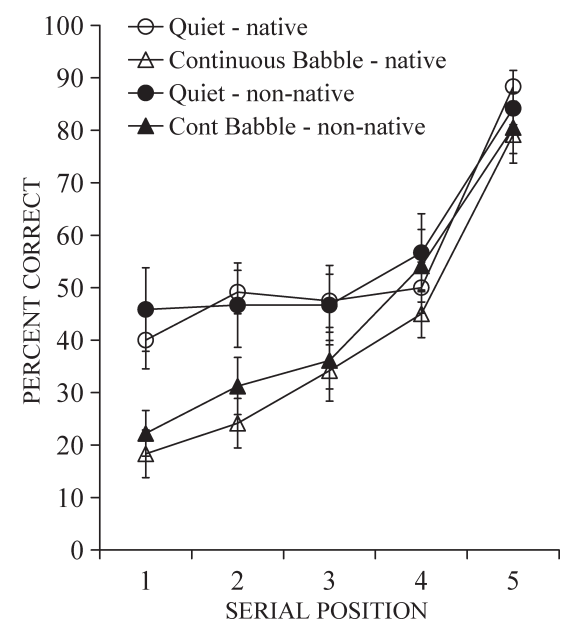

Figure 1. Average percentage correct as a function of serial position for word pairs presented in quiet (circles) and continuous babble (triangles). Open symbols indicate results from the original Murphy et al. (2000) study with native English speakers; filled symbols indicate the replication with non-native English speakers. Vertical bars depict standard errors of the means. performance, nor did it interact with either serial position, or the presence versus absence of noise $($ all $F \mathrm{~S}<1)$. These results indicate that the effect that babble has on the early serial positions is robust and that it generalizes to proficient nonnative speakers of English. Because there were no differences in the effect that babble had on memory performance between native and nonnative speakers, we collapsed over the results of both language groups to increase the statistical power of our two main comparison conditions: memory performance in quiet and in continuous babble. In the remainder of the paper, we use these combined data when comparing memory between new experimental conditions and memory in quiet $(n=30)$ and in continuous babble $(n=33)$.

\section{Design and hypotheses}

We conducted four experiments to investigate the degree to which (a) perceptual degradation, (b) cognitive level interference due to involuntary processing of irrelevant material, and/or (c) the diversion of attentional resources to facilitate stream segregation affect memory when babble is continuously present. In Experiment 1 we presented babble only during word pair presentations. In Experiment 2 the babble was presented only during the rehearsal period between word pair presentations. In Experiment 3, the babble started $500 \mathrm{~ms}$ before the presentation of a word pair and ended $500 \mathrm{~ms}$ after the end of the word pair. A schematic representation of these three conditions is displayed in Figure 2. We also employed a divided-attention paradigm (Experiment 4) to directly test the notion that continuous babble engaged attention-demanding top-down processes. We suggest that if it is the perceptual distortion of the words per se that is responsible for the memory decrements in the early serial positions, then presenting the babble only when the words are presented will achieve the same degree of energetic masking and therefore will lead to the same decrease in memory performance. However, if it is the involuntary processing of background babble at a phonological, semantic, 


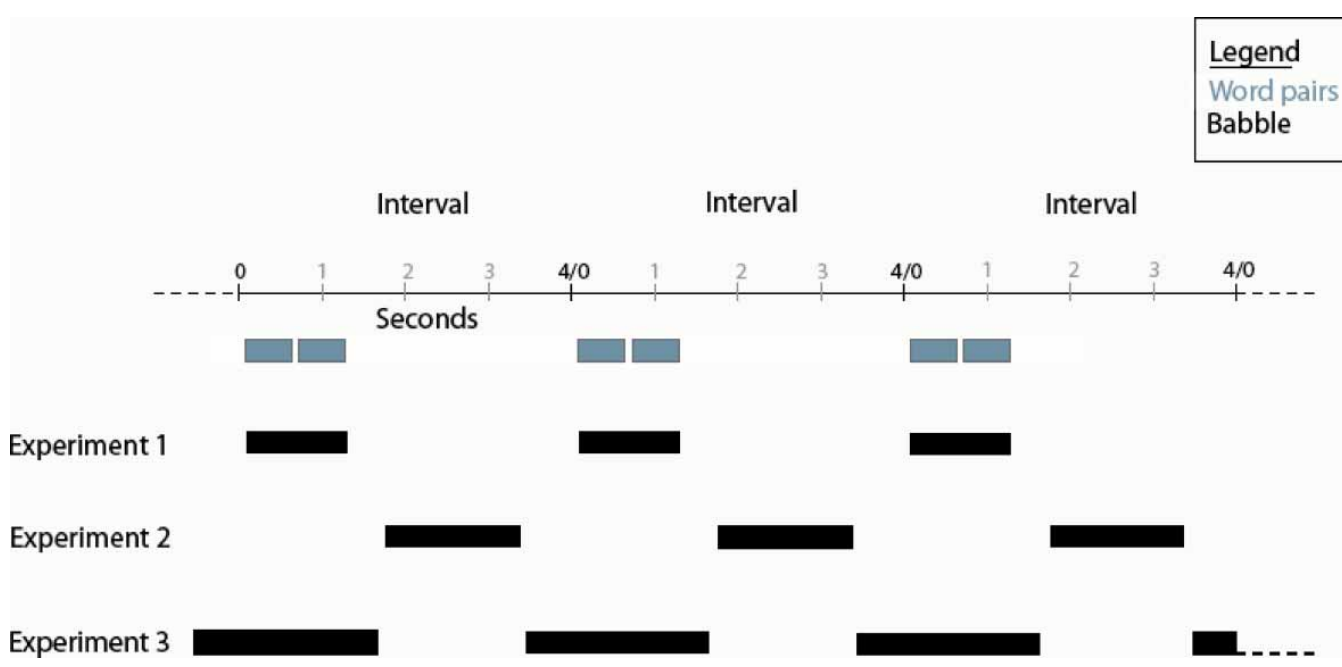

Figure 2. Stimulus conditions in Experiments 1-3.

and/or linguistic level that is disrupting encoding during the rehearsal period between word pairs, we should be able to generate equivalent memory deficits when presenting the babble only between stimulus pairs - that is, during the period in which rehearsal and encoding (but not word recognition) occurs. Alternatively, if it is the diversion of attentional resources to facilitate auditory streaming that adversely affects memory under conditions of continuous babble, we would expect to see a decline when babble is switched on shortly before the onset of the word pairs.

\section{General methods}

The stimuli, apparatus, testing protocols, subject pools, and research technicians were identical between Murphy et al. (2000), the replication using fluent ESL participants, and the following experiments. Hence any differences between the present results and those found in Murphy et al. and its replication cannot be attributed to differences in any of these factors.

\section{Participants}

A total of 99 undergraduate students at the University of Toronto were paid $\$ 10$ per hour for participating in Experiments 1 to 4. Details concerning age, gender, years of education, and Mill Hill vocabulary score for all four experiments are presented in Table 1. All participants were required to have clinically normal hearing (Clark, 1981) over the speech range as measured by airconduction pure-tone thresholds. Additionally,

Table 1. Participant parameters for Experiments 1-4

\begin{tabular}{|c|c|c|c|c|c|c|c|c|c|c|}
\hline \multirow[b]{2}{*}{ Experiment } & \multirow[b]{2}{*}{$N$} & \multirow[b]{2}{*}{ Age range $^{\mathrm{a}}$} & \multicolumn{2}{|c|}{$A g e^{\mathrm{a}}$} & \multicolumn{2}{|c|}{ Gender } & \multicolumn{2}{|c|}{ Education $^{\mathrm{a}}$} & \multicolumn{2}{|c|}{ Vocab. score ${ }^{\mathrm{b}}$} \\
\hline & & & $M$ & $S D$ & Female & Male & $M$ & $S D$ & $M$ & $S D$ \\
\hline 1 & 16 & $18-23$ & 19.69 & 1.35 & 12 & 4 & 15.06 & 1.88 & 13.38 & 1.63 \\
\hline 2 & 15 & $18-24$ & 20.13 & 1.55 & 13 & 2 & 15.67 & 1.72 & 14.47 & 1.51 \\
\hline 3 & 15 & $18-24$ & 20.60 & 1.81 & 9 & 6 & 15.13 & 1.55 & 13.80 & 2.68 \\
\hline $4 \mathrm{~A}$ & 32 & $18-25$ & 20.88 & 1.52 & 17 & 15 & 15.87 & 1.52 & 13.53 & 2.38 \\
\hline $4 \mathrm{~B}$ & 21 & $18-25$ & 20.34 & 1.66 & 25 & 7 & 15.00 & 1.85 & 13.94 & 2.26 \\
\hline
\end{tabular}

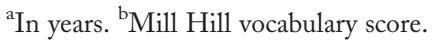


measures of visual acuity (Snellen fraction) were collected in Experiment 4 because participants performed a visual secondary task. Visual acuity was measured binocularly (for a $20-\mathrm{ft}$ distance) and did not vary for the participants in Experiments $4 \mathrm{~A}$ and $4 \mathrm{~B}(M=22.30, S D=$ 7.42 , for participants in Experiment $4 \mathrm{~A}$ and $M$ $=19.00, S D=6.43$ for those in Experiment 4B). Note that in Experiment 4A only 10 out of the 32 participants contributed data to the visual acuity measurement because it was obtained at a later point in time. The remaining participants reported normal or corrected-to-normal vision.

\section{Apparatus and stimuli}

The word pairs, which were the same as those in Murphy et al. (2000), consisted of 400 two-syllable common nouns with a frequency of more than 1 per million (Kucera \& Francis, 1967). The individual words, spoken by a female speaker, were digitally recorded at a sampling rate of $20 \mathrm{kHz}$ using the Computerized Speech Research Environment (CSRE; AVAAZ Innovations, 1994) software. All recordings had similar root-mean-square (RMS) values. The word pairs were delivered through a 16-bit digital-to-analog converter (TDT DD1) followed by a $10-\mathrm{kHz}$ low-pass filter (TDT FT6-2, 60-dB attenuation at $11.5 \mathrm{kHz}$ ), a programmable attenuator (TDT PA4), and a weighted signal mixer (TDT SM3) to the left side of TDH-49 earphones. All testing took place in a doublewalled sound-attenuating chamber.

\section{Procedure}

Babble threshold. The babble materials used in these experiments were taken from the Revised Speech Perception in Noise (R-SPIN) test (Bilger, Nuetzel, Rabinowitz, \& Rzeczkowski, 1984). Thresholds for the detection of 12 -talker babble were determined for each individual using an adaptive two-interval, two-alternative forced-choice paradigm. A trial consisted of the sequential presentation of two intervals of $1.5 \mathrm{~s}$ duration, separated by a 1.5 -s silent period between intervals. Trials were initiated by pressing the central button on a response box containing three buttons, with the first interval starting $1.5 \mathrm{~s}$ after the button press. The occurrence of the first interval was signalled by illuminating a light-emitting diode (LED) above the leftmost button on the response box; a second LED above the rightmost button was illuminated during the second interval. A segment of babble was assigned randomly to one of the two intervals, and the participant indicated which interval contained the babble segment by pressing the appropriate response button. Feedback was provided by flashing the LED corresponding to the interval in which the babble segment occurred. The starting intensity was $50 \mathrm{~dB}$ SPL. The intensity of the babble was reduced after three correct responses in a row and increased after a single incorrect response, an adaptive procedure that estimates the $79 \%$ point on the psychometric function (Levitt, 1971). The session was terminated after 12 reversals (change from decreasing to increasing intensity level or vice versa). The babble threshold was defined as the average SPL on the last eight reversals. Babble thresholds were determined for the right ear first. Because stimuli were only presented to the left ear during these experiments, only left-ear thresholds are shown in Table 2. In all experiments reported here, the words were presented at a level that was individually set to $50 \mathrm{~dB}$ above that listener's babble threshold.

Individually adjusting the signal-to-noise ratio. The low-context sentences from the R-SPIN test (Bilger et al., 1984) were used to determine the

Table 2. Babble and SPIN thresholds in the left ear for Experiments 1-4

\begin{tabular}{|c|c|c|c|c|c|}
\hline \multirow[b]{3}{*}{ Experiment } & \multirow[b]{3}{*}{$N$} & \multicolumn{4}{|c|}{ Left-ear threshold } \\
\hline & & \multicolumn{2}{|c|}{ Babble } & \multicolumn{2}{|c|}{ SPIN } \\
\hline & & $d B S P L$ & $S D$ & $S N R$ in $d B$ & $S D$ \\
\hline 1 & 16 & 15.30 & 2.92 & 0.44 & 2.25 \\
\hline 2 & 15 & 15.34 & 3.20 & 1.93 & 1.86 \\
\hline 3 & 15 & 16.90 & 1.08 & 1.79 & 1.73 \\
\hline $4 \mathrm{~A}$ & 32 & 16.14 & 5.26 & & \\
\hline
\end{tabular}


SNR for each individual that resulted in 50\% correct identification of the sentence final words, following the procedure described in Murphy et al. (2000). In short, each participant listened to at least two R-SPIN lists at SNRs that were chosen to bracket the $50 \%$ intelligibility point for the final words in low-context sentences. The SNR corresponding to the 50\% point was then determined by linear interpolation between these two values. This individual SNR value, lowered by another $7 \mathrm{~dB}$, was then used in the memory task. Equating for individual differences in SNR in this way made it equally hard for all listeners to hear the words presented in babble and was found by Murphy et al. to result in a word identification accuracy of $91 \%$. Table 2 presents the average SNR for $50 \%$ intelligibility of a low-context sentence in Experiments $1-3$. The average SNR in the Murphy et al. study (Experiment 3$)$ was $-0.13 \mathrm{~dB}(S D=1.25)$.

Word recall. In Experiments 1 to 4 participants listened to the words that were randomly arranged in 40 lists containing five word pairs each following the paradigm by Madigan and McCabe (1971). The words were randomly paired, and any obvious association between two words was avoided. Four seconds after a short warning tone $(1 \mathrm{kHz}$ at $90 \mathrm{~dB}$ SPL for $500 \mathrm{~ms})$ had indicated the beginning of a list, the first word pair was presented with a silent period of $100 \mathrm{~ms}$ between the words. Word pairs were played at a rate of 1 pair every four seconds. Four seconds after the start of the final word pair a short warning tone indicated the beginning of the recall phase. After another four seconds participants were cued with the first word from one of the five previously presented word pairs and were asked to recall the second word of the pair. Only one pair from each list was cued; no time limit was placed on recall, and participants were encouraged to guess.

The first serial position refers to the first word pair and so forth. List order was identical for all participants, and the serial position of each word pair within the five-word-pair list was tested an equal number of times (eight times) within a session. The order in which the serial positions were tested was independently and randomly determined for each participant. There were no practice trials, and no feedback was provided. There was a break after the presentation of the first 20 lists.

The word pairs were presented at a sensation level of $50 \mathrm{~dB}$. The 12-talker babble taken from the R-SPIN test was, when present, set so as to produce a SNR of $-7 \mathrm{~dB}+$ the individual's SPIN threshold.

\section{EXPERIMENT 1}

Experiment 1 investigated whether the memory deficit was caused by energetic masking of the target words. To test this hypothesis, the babble noise was gated on and off with each word pair in such a way that it started simultaneously with the onset of the first word of the pair and finished with the offset of the second word. The time period between the offset of the second word of the previous pair and the onset of the next word pair was filled with silence (Figure 2). Because the SNR was individually adjusted following the procedure employed by Murphy et al. (2000), the amount of energetic masking during stimulus presentations, and therefore the amount of perceptual distortion induced by the presence of noise, should be comparable between Murphy et al.'s continuous and the present discontinuous babble. If the memory deficit for continuous babble was due to perceptual distortion, we should find the same (or greater) decrement when the babble is gated on and off with the words, because word recognition is poorer when the masker is gated on and off with the word than it is when the masker is continuously present (Wagener \& Brand, 2005). On the other hand, if either the resource demands of tracking the babble to facilitate perceptual streaming or the involuntary processing of babble noise during the interword interval are responsible for the memory declines in continuous babble, then we should find little or no decline in memory in Experiment 1, compared to performance in quiet. 


\section{Results and discussion}

The percentage of words correctly remembered at each serial position served as the dependent measure. Figure 3 compares memory performance for listeners in the current experiment (WordBabble) with memory for the word pairs tested in quiet and continuous babble. The last two conditions combined results from Murphy et al. (2000) and the replication using fluent ESL participants. In the Quiet condition, the word pairs were presented at $65 \mathrm{~dB} \mathrm{SPL}$ in a quiet background. In the Continuous Babble condition, the same word pairs were presented in a background of individually adjusted continuous babble at $50 \mathrm{~dB}$ SL. In the present experiment, the word pairs were presented at $50 \mathrm{~dB}$ SL in equally adjusted discontinuous babble that was only present during word pair presentations. Figure 3 shows very similar recall for the Quiet condition and the discontinuous Word-Babble condition. Only continuously present babble led to lower recall, especially in the first two serial positions. A 3 (perceptual manipulation: quiet, continuous babble, word-babble) by 5 (serial

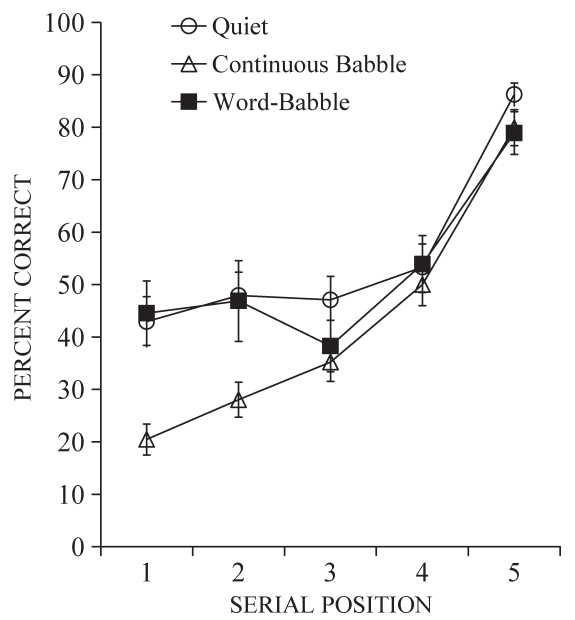

Figure 3. Average percentage correct as a function of serial position for word pairs presented in quiet (open circles), continuous babble (open triangles), and discontinuous babble presented only during word presentations (word-babble, filled squares). Vertical bars depict standard errors of the means. position) mixed-measures ANOVA revealed the following effects: a main effect of serial position, $F(4,304)=88.49, M S E=279.19, p<.0001$, a main effect of perceptual manipulation, $F(2,76)$ $=5.41, M S E=1,265.43, p=.006$, and an interaction between the two factors, $F(8,304)=3.24$, $M S E=279.19, p=.001$. When the number of perceptual manipulation conditions was reduced to two by removing the continuous babble condition, a 2 (perceptual manipulation: quiet, word-babble) by 5 (serial position) mixedmeasure ANOVA showed a significant effect of serial position, $F(4,176)=41.64, M S E=$ $274.20, p<.0001$, but no effect of manipulation, $F(1,44)<1, M S E=1,531.82, p=.58$, and no interaction, $F(4,176)<1, M S E=274.20$, $p=.49$. Hence, the serial position by manipulation interaction was due to the presence of the continuous babble group.

In summary, the results of Experiment 1 suggest that the effect of continuous babble on memory performance cannot be attributed solely to deficiencies in the perceptual representation of the words. Even though the SNR was individually adjusted in the same way for both the Continuous and the Word-Babble conditions (so that perceptual degradation should be approximately the same), the former but not the latter experiment yielded a drop in memory performance.

\section{EXPERIMENT 2}

Experiment 2 investigated whether the presence of babble between stimulus presentations led to the observed memory deficit, possibly by interfering with rehearsal and subsequent consolidation of items in long-term memory. Therefore, babble was presented only between word pairs, and not during the presentation of the word pairs Between-words Babble). The babble noise was gated on and off between word pairs, starting $500 \mathrm{~ms}$ after the end of a word pair and terminating $500 \mathrm{~ms}$ before the beginning of the next word pair (Figure 2). 


\section{Results and discussion}

Figure 4 plots percentage correct as a function of serial position when there was no masker (Quiet), when the masker was a continuous babble background, and with Between-words Babble. The results show that there is no difference in recall performance between the Quiet and Discontinuous Babble conditions. Figure 4 also suggests that only the continuous babble condition led to lower recall, especially in the first two serial positions. A 3 (perceptual manipulation: quiet, between-words babble, continuous babble) by 5 (serial position) mixed-measures ANOVA revealed main effects of serial position, $F(4,300)$ $=95.94, M S E=288.89, p<.0001$, and background condition, $F(2,75)=5.47, M S E=$ 1,292.97, $p=.006$, and an interaction between the two, $F(8,300)=2.28, M S E=659.70$, $p=.02$. Again, removing the continuous babble condition from the ANOVA eliminated both the effect of condition as well as the interaction effect: A 2 (perceptual manipulation: quiet, between-words babble) by 5 (serial position) mixed-measure ANOVA showed only a

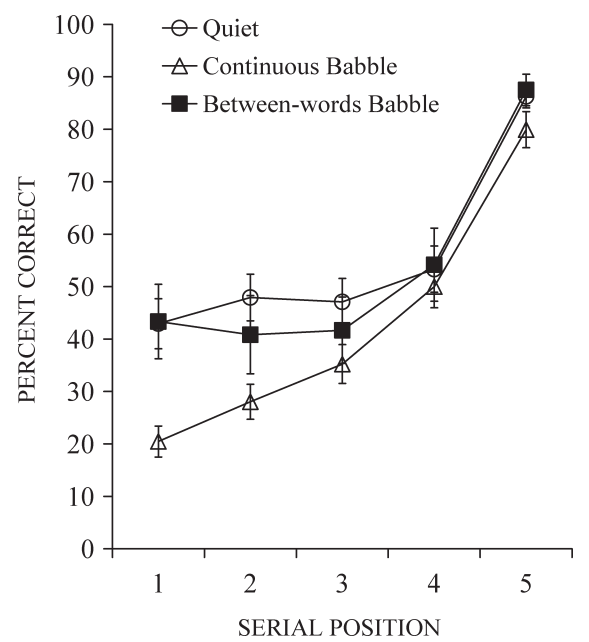

Figure 4. Average percentage correct as a function of serial position for word pairs presented in quiet (open circles), continuous babble (open triangles), and discontinuous babble presented between word presentations (filled squares). Vertical bars depict standard errors of the means. significant effect of serial position, $F(4,172)=$ 47.53, $M S E=290.99, p<.0001$, and not of condition, $F(1,43)<1, M S E=1,586.05, p=.72$, and no interaction, $F(4,172)<1, M S E=$ 290.99, $p=.71$. Hence, presenting babble between word pairs did not affect memory encoding.

According to the interference hypothesis, we would have expected the presence of babble between word presentations to have had a detrimental effect on encoding and rehearsal. Given that this result did not occur, either the babble presented between words did not activate phonemic and/or semantic processes, or if it did, this activation is not responsible for the adverse effects of continuous babble.

\section{EXPERIMENT 3}

The results of Experiments 1 and 2 showed that the presence of a masker only during word presentations (Experiment 1), or only between word presentations (Experiment 2), had no effect on memory performance, whereas the presence of a masker both during and between word presentations (Continuous Babble) did. Hence, the effect of continuous babble must be related either to its continuity throughout the trial, or to its presence before, during, and after word presentations. To investigate which of these two possibilities was responsible for the memory effect in Experiment 3, we gated the masker on $500 \mathrm{~ms}$ before the onset of the first word in a pair and gated it off $500 \mathrm{~ms}$ after the last word in a pair (Discontinuous Babble). If the masker had to be continuous in order to produce a reduction in memory, we would expect memory performance in Experiment 3 to be identical to that in quiet. If, on the other hand, the presence of the masker before, during, and after word pair presentation was responsible for the memory effect, we would expect to find a decrement in memory performance in Experiment 3 that was equivalent to that found in a continuous masker. 


\section{Results and discussion}

Figure 5 plots percentage correct as a function of serial position under conditions when no background babble was present, when the babble was continuous, and with discontinuous babble. In contrast to Experiments 1 and 2, memory performance in discontinuous babble is similar to that observed in the Continuous Babble condition and different from performance in the quiet baseline. A mixedmeasures ANOVA with perceptual manipulation (quiet, discontinuous babble, continuous babble) as a between-subjects variable and serial position as a within-subject variable showed a main effect of serial position, $F(4,300)=93.81, M S E=$ 290.68, $p<.0001$, and perceptual manipulation, $F(2,75)=6.79, M S E=1,141.36, p=.002$. The interaction effect also reached significance, $F(8$, $300)=2.04, M S E=290.68, p=.04$. When the quiet baseline condition was removed from the analysis, the effect of perceptual manipulation, $F(1,46)<1, M S E=907.92, p=.89$, and the interaction, $F(4,184)=1.42, M S E=301.62$, $p=.23$, disappeared. Only the serial position effect remained significant, $F(4,184)=61.12$, $M S E=301.62, p<.0001$.

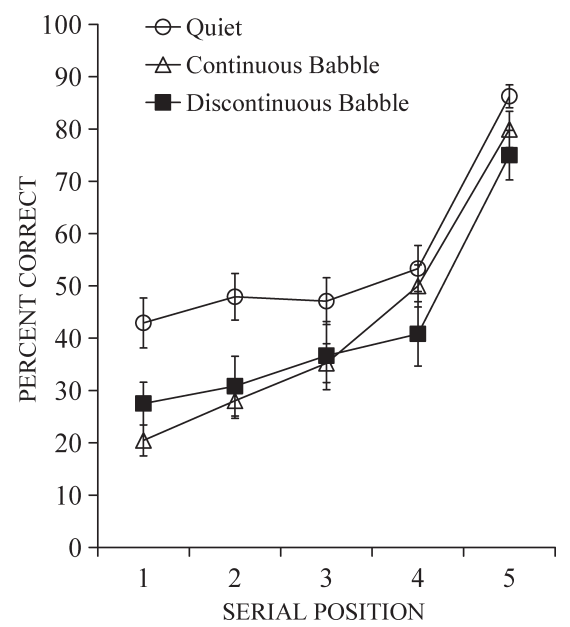

Figure 5. Average percentage correct as a function of serial position for word pairs presented in quiet (open circles), continuous babble (open triangles), and discontinuous babble gated on 500 ms before the onset and off $500 \mathrm{~ms}$ after the offset of the word pairs (filled squares). Vertical bars depict standard errors of the means.
There are two possible reasons why presenting the masker before, during, and after word pair presentations could produce a memory decrement on the order of that observed for a continuous masker. The first has to do with the perceptual degradation hypothesis, the second with auditory streaming.

\section{Perceptual degradation due to forward and backward masking}

In Experiment 1, masking occurred only during word pair presentations, whereas in the continuous-masking condition, the masker was present before, during, and after word pair presentations. It is possible that the combined effects of forward, simultaneous, and backward masking on word recognition are greater than those produced by simultaneous masking alone. Hence we might expect a greater amount of perceptual degradation in Experiment 3 than in Experiment 1. However, several results in the masking literature argue against this interpretation. First, various studies (McFadden \& Wright, 1990; Wright, 1997; Zwicker, 1965) have shown that detection of sinusoidal stimuli as well as narrow-band noise is better when the masker is continuous than when it is gated on and off with the signal. Hence, adding a forward and backward component to a simultaneous masker improves rather than degrades detection. Second, there is some evidence that word recognition is better when the masker is continuous than it is when the masker is gated on and off with the words. Wagener and Brand (2005) measured speech intelligibility for short sentences presented in speech-shaped noise that was either on continuously or gated on and off with the sentences. They found that the SNR required for $50 \%$ word recognition was lower when the masker was continuous than when the masker was gated on and off with the sentences. This is the opposite of what we would expect if the addition of forward- and backward-masking components increased the amount of masking (perceptual degradation). Hence, it is unlikely that the memory decrement observed in Experiment 3 was due to an increase in perceptual degradation. 


\section{Stream segregation}

If the memory deficits observed in Experiment 3 and in continuous babble are not due to perceptual degradation, or to phonemic or semantic interference, then the presence of the babble before and after word presentations must be engaging other processes that do interfere with memory. We propose that continuous babble interferes with memory because the participant is attending to the auditory stream in order to enhance word detection and recognition.

We have already seen that a signal is easier to detect (McFadden \& Wright, 1990; Wright, 1997; Zwicker, 1965), and speech is more easily recognized (Wagener \& Brand, 2005) when the masker is continuous than when it is gated on and off with the signal. Both of these effects are likely to be a consequence of auditory streaming. Bregman (1990) has argued that when the auditory scene contains multiple sound sources, the listener engages a number of perceptual and cognitive mechanisms to identify and segregate the different sources of sound. Moreover, successful sound segregation has been shown to lead to better speech recognition (for a review, see Schneider, Li, \& Daneman, in press). Hence, if the presence of the babble masker before and/or after word presentations facilitates stream segregation, we would expect these mechanisms to be engaged earlier and more effectively in Experiment 3 and in continuous babble than when the babble is gated on and off with the words. Carlyon, Cusack, Foxton, and Robertson (2001) have shown that stream segregation requires some time to build up. Presenting the babble prior to word onset affords the auditory system the opportunity to focus attention on the babble, which, in turn, would make it easier to segregate the words from the babble background. When, however, the masker is gated on and off with the words, it may take longer to segregate the words from the babble background because stream segregation is not instantaneous (Carlyon et al., 2001). Indeed it is possible that most of the word will have been presented by the time stream segregation is in place. Hence, there is reason to believe that the perceptual and cognitive processes involved in stream segregation will be engaged earlier, persist longer, and be more effective when the masker precedes and follows the signal.

Why would the earlier and more persistent engagement of streaming mechanisms have an adverse effect on memory performance? One possible answer is that sound segregation is an attention-demanding process and that it is these demands on attention that lead to memory decrements. A number of studies, using a variety of paradigms, have demonstrated that streaming can indeed be attention-demanding. For instance, Carlyon et al. (2001) used ABA sequences of tones to show that the proportion of times the stimuli were judged to fall into two streams was reduced when attention was diverted to a competing task. Alain and Izenberg (2003), using event-related potentials (ERPs) in an oddball paradigm, found a similar result. They measured mismatch-negativity $(\mathrm{MMN})$ responses to tuned and mistuned tones in the unattended ear while asking participants to perform two differentially difficult auditory tasks on the sound in the other, attended, ear. They found that the amplitude of the MMN elicited by the mistuned sound in the unattended ear decreased with increasing task demand of the auditory task within the attended ear. They argued that the MMN response in their task was reduced because the more difficult auditory task put a greater demand on a fixed pool of resources, leaving fewer resources available for processing events occurring outside the "spotlight" of attention (i.e., in the unattended ear).

These results are consistent with an interpretation within the framework of the limitedresource model (Craik \& Byrd, 1982). This model suggests that age, divided attention, and other manipulations reduce the resources that are available to encode information. If attending to the auditory stream in order to enhance detection and recognition of words draws on the same limited pool of resources as do memory encoding and rehearsal processes, then we would expect the presence of continuous babble to be deleterious to memory. However, when the babble is only intermittently present so that it does not afford 
the same opportunity to facilitate stream segregation (babble gated on and off with the words), or when the babble is absent during word presentations (babble between word pairs) so that attentional resources are not required for stream segregation, babble should have little effect on memory performance because attentional resources remain available for encoding and rehearsal.

If perceptual streaming engages attentional resources on a more central or cognitive level of processing, then any other competing task that engages attentional resources at this level should produce memory deficits comparable to those that are observed when the babble noise precedes and follows word presentations. Moreover, if both continuous noise and the secondary task only affect performance at a cognitive level, it should not matter whether the stimuli employed in the secondary task are presented visually, rather than aurally. In other words, the amount of interference produced by the secondary task should be modality-independent and depend only on task difficulty, as nicely illustrated in a psychophysical study conducted by Bonnel and Hafter (1998). Bonnel and Hafter found that when the task demand was high (identifying which of two stimuli was presented), performance trade-offs were observed between the primary and secondary tasks. However, when the same stimuli were used in the competing task, but the task demands were lowered (detect the presence of a stimulus), performance trade-offs disappeared. The same pattern of results was observed for the identification task when the stimuli belonged to different modalities (auditory and visual). They suggested that stimulus modality was less important as a determinant of performance because task interference primarily occurred at central processing levels.

The limited-resources theory suggests that the success of memory recall depends on the depth and elaborateness of encoding, which, in turn, depends on the amount of central resources available for the encoding process. Words are most likely to be encoded in a deep and elaborate fashion when there are no competing demands on this pool of resources (Craik \& Byrd, 1982; Craik, Govoni, Naveh-Benjamin, \& Anderson, 1996; Naveh-Benjamin, 2001; Rabinowitz, Craik, \& Ackerman, 1982). Conversely, when resources are more limited for memory encoding because, for instance, a secondary task is added, memory recall suffers because the qualitative nature of the encoding changes, and individuals learn words in a less deep or semantic manner (Anderson \& Craik, 1974; Craik \& Byrd, 1982). Moreover, earlier list positions are more adversely affected than later positions because retrieval of more recently presented items (late serial positions) is supported by transient acoustic or phonological information, whereas retrieval of early items relies on semantic information, whose encoding may be disrupted by the diversion of central resources to stream segregation. We propose that listening under conditions that require central resources to achieve stream segregation is somewhat akin to a dual-task situation because the participant must simultaneously monitor the auditory input and rehearse and encode previously heard words.

\section{EXPERIMENT 4}

In Experiment 4, we compared memory performance for continuous background babble to memory performance for a simultaneously presented visual digit-monitoring task in which participants were asked to monitor a stream of single digits for the occurrence of three successive odd digits. In Experiment 4A one group of participants encoded word pairs for later recall while simultaneously monitoring a sequence of visually presented digits. In Experiment $4 \mathrm{~B}$ a second group carried out the digit-monitoring task alone. We wish to argue that continuous babble and a secondary task are functionally equivalent and therefore should result in the same pattern of results because both put additional attentional demands on the participant performing the memory task. The digit-monitoring secondary task was assumed to divert some of the processing resources away from the auditory memory task and has been 
shown to be effective in reducing performance on primary memory tasks (Mangels, Craik, Levine, Schwartz, \& Stuss, 2002; Troyer \& Craik, 2000).

\section{Secondary task}

The secondary task was a visual digit-monitoring task in which participants monitored a computer screen for the consecutive appearance of three odd digits (e.g., 3-7-1, 9-5-9). Participants signalled detection of targets by pressing the left mouse button. Yellow digits were presented one at a time on a blue background. Recall that the primary task consisted of the auditory presentation of five word pairs and lasted for approximately 20 s. The secondary task was presented with each memory task trial in Experiment 4A and by itself in Experiment 4B. Each secondary task trial started $4 \mathrm{~s}$ before the first word pair was presented and lasted for $26 \mathrm{~s}$. Digits on a given trial were presented at one of four possible presentation speeds, one digit every $2.6,1.3,1.0$, or $0.5 \mathrm{~s}$. Hence, from the slowest to the fastest presentation rate, 10, 20, 26 , or 52 digits were presented per trial. Because a target was defined as the occurrence of three consecutive odd digits, the maximum number of possible targets was 3 at the slowest presentation rate, 6 at the next faster rate, 8 at the second fastest rate, and 17 at the fastest presentation rate. The secondary task was designed in such a way that regardless of how many targets were theoretically possible, in practice each trial contained 0 , 1 , or 2 targets. The number of actual targets in a given trial was varied at random. When there were 2 targets in the trial, the targets were chosen such that the first target appeared within the first $13 \mathrm{~s}$ of the trial and the second target within the second $13 \mathrm{~s}$ of the trial. A short training session on the digit-monitoring task alone was administered to all participants, giving them a chance to familiarize themselves with the task and the different presentation speeds.

In the dual task, the speed of the digits presented on a word pair trial and the list position tested at recall were independently and randomly assigned on each trial, subject to the constraints that each serial position must be tested 8 times, and each presentation speed must occur 10 times during a session. This procedure was followed to prevent a systematic relationship between presentation speed and serial position tested in the subsequent recall. In the fullattention digit task, the speed of digit presentation and number of targets were also randomized for each volunteer.

\section{Results and discussion}

Figure 6A plots the divided-attention memory performance in Experiment 4A, collapsed across rate of digit presentation, ${ }^{1}$ together with the Quiet baseline and Continuous Babble conditions. The figure shows that recall under divided attention (DA) is reduced compared to the (fullattention) baseline condition, especially in the first four serial positions. In this regard, the pattern looks similar to recall in continuous babble where recall is also most affected in early serial positions. Comparing recall under DA and babble directly, the plot shows that the adverse effect of continuous babble on memory is considerably stronger and more pronounced for the early serial positions than is the effect of the DA task. On the other hand, looking at Figure 6B, the effect of discontinuous babble (Experiment 3 ) is very similar to the effect of DA. A 2 (attention condition: DA vs. full-attention baseline) by 5 (serial position) mixed-measures ANOVA showed main effects of serial position, $F(4,240)$ $=72.10, M S E=316.58, p<.0001$, and attention condition, $F(1,60)=4.79, M S E=1,159.21$, $p=.03$, indicating that recall under DA was significantly lower than that in the baseline, fullattention condition. The interaction between the two factors was not significant, $F(4,240)<1$, $M S E=316.58, p=.54$. A second ANOVA that compared recall under DA with recall under continuous babble yielded a main effect for serial

\footnotetext{
${ }^{1}$ Preliminary statistical analyses found no statistically significant effect of presentation rate, possibly because presentation rate was randomized within a list.
} 


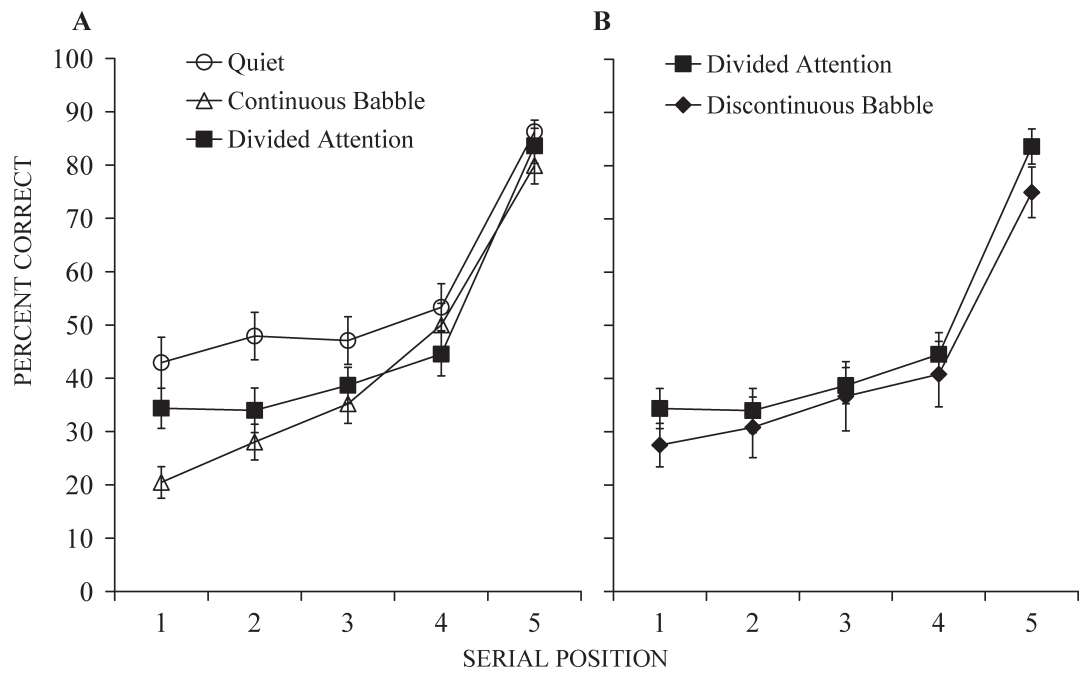

Figure 6. (A) Average percentage correct as a function of serial position for word pairs presented in quiet (open circles), in continuous babble (open triangles), and under divided attention (filled squares). (B) Average percentage correct as a function of serial position for word pairs presented with preceding babble (Experiment 3, diamonds) and under divided attention (Experiment 4, squares). Vertical bars depict standard errors of the means.

position only, $F(4,252)=97.49, M S E=364.85$, $p<.0001$ (adjusted for violations of the assumption of homogeneity). The main effect of condition (DA vs. babble) was not significant, $F(1$, $63)=1.74, M S E=864.88, p=.19$. However, the interaction approached significance, $F(4$, $252)=2.43, M S E=364.85, p=.06$. Finally, repeating the latter ANOVA but substituting discontinuous babble from Experiment 3 for continuous babble removed even the hint of an interaction, $F(4,180)<1, M S E=350.88$, $p=.93$. The results indicate that continuous babble had a marginally stronger effect on memory than DA on the first two serial positions, whereas discontinuous babble matched DA performance very closely. The results of this experiment indicate that attending to the auditory stream in a memory task is at least as demanding as simultaneously monitoring a series of visually presented digits where the rate of digit presentation varies from trial to trial.

Two 4 (presentation speed: 2.6, 1.3, 1.0, $0.5 \mathrm{~s}$ per digit) by 2 (attention condition: full or divided attention) mixed-measures ANOVAs, conducted on the performance measures of the digit-monitoring task (detection accuracy and reaction time), revealed the expected main effect of attention: Participants performed consistently better when the monitoring task was performed alone than when attention was divided between digit monitoring and memory encoding: detection accuracy, $F(1,51)=54.33, M S E=711.87$, $p<.001$; RT, $F(1,51)=14.03, M S E=0.045$, $p<.001$. Additionally, both conditions showed an effect of presentation speed indicating that faster presentation rates led to greater accuracy, $F(3,153)=4.00, M S E=133.65, p<.01$. Post hoc tests (Bonferroni adjusted) indicated that the effect was carried by a significant increase in accuracy between the slowest and the fastest presentation rates. Neither accuracy nor RT revealed an interaction with attention.

Experiments 4A and 4B indicate that the digitmonitoring task adversely affected memory, and vice versa. This result fits well with a large body of evidence showing that performance in a primary (memory) task is reduced considerably when carried out simultaneously with a secondary task (e.g., Craik et al., 1996; Rabinowitz et al., 1982). Typically this result is interpreted to show 
that primary and secondary tasks share attentional and/or processing demands.

The memory deficits in auditory streaming conditions (continuous and discontinuous preceding babble) are very similar in shape and size to the memory deficits seen in Experiment 4, which used a more conventional dual-task paradigm. Based on the fact that performing the memory task in continuous background babble and under dual-task conditions produces similar results, we suggest that it is plausible to assume at least some functional commonalities in the underlying processes. Specifically, we propose that attending to the auditory stream and encoding the words into memory both require attention, and, because attention is limited, auditory streaming affects memory performance. Therefore, this series of experiments is consistent with the hypothesis that participants, when listening to aurally presented word pairs in a continuous background noise, are compelled to attend to the auditory stream in order to segregate the target words from the background. Attentional resources that otherwise would be dedicated to encoding and rehearsal when attempting to memorize the list are diverted to focus attention on the auditory stream. As a result, memory performance suffers.

\section{GENERAL DISCUSSION}

Murphy et al. (2000) showed that memory performance in an auditory paired-associate memory task was impaired when words were presented in a background babble. We investigated three possible explanations for this effect. In Experiment 1 we investigated whether the babble masker led to a distorted perceptual representation of the word, compromising higher-level memory processes and leading to declines in recall performance. Experiment 2 looked at whether the presence of babble between word pair presentations initiated activity in the phonological, semantic, and/or linguistic systems that could not be inhibited and therefore interfered with rehearsal and encoding. Experiment 3 examined whether the presence of babble before, during, and after word pair presentations required attentional resources to be deployed to monitor the auditory scene and enhance the detection and recognition of words. Finally, Experiment 4 was added to see whether a nonauditory, attention-demanding task would produce results similar to those found in Experiment 3.

The pattern of results in Experiments 1 and 2 failed to support either the perceptual distortion hypothesis or the notion that babble interferes with memory because the babble elicits activity that interferes with encoding and rehearsal. The results of Experiment 3 indicated that when the babble was turned on early enough to allow some stream segregation to build up, memory performance was almost the same as that observed when the babble was continuous. Taken together, the results from Experiments 1 to 3 are consistent with the hypothesis that in the presence of surrounding babble the listener closely monitors the auditory input to enable her or him to segregate the target words from the babble background.

In Experiment 4, we showed that the addition of a secondary task, delivered in a different modality, produced a similar pattern of memory deficits as that found for continuous babble. Because the secondary task was visual, it had to be affecting memory performance at a more central cognitive level. Hence these results are consistent with the hypothesis that the reason for memory decrements in the early serial positions is that monitoring the auditory stream for the word pairs to segregate them from the background draws upon central attentional resources and that the ensuing reduction in resources adversely affects encoding processes such as rehearsal, elaboration, and the formation of associations. In that sense, attending to the auditory stream is equivalent to performing a secondary task while attempting to rehearse and encode the words.

Interestingly, memorizing in a Continuous Babble background was even more difficult than memorizing while simultaneously engaging in a digit-monitoring task. The implication of this finding is that attempting to comprehend and remember material in noise is likely to have a much larger effect in everyday situations than 
previously realized. Normally, the effects of noise are evaluated in terms of its effect on word recognition. The present results, and those of $\mathrm{McCoy}$ et al. (2005), Murphy et al. (2000, 2006), Schneider et al. (2000), and Rabbitt (1968, 1991), indicate that noise can have an adverse impact on comprehension and memory even when word identification performance is good (approximately 90\% in the present case). Hence, in evaluating the adverse effects of noise on performance, we need to consider how noise affects higher order cognitive processes, not just how it affects word recognition.

Original manuscript received 5 June 2006

Accepted revision received 2 April 2007 First published online 27 July 2007

\section{REFERENCES}

Alain, C., \& Izenberg, A. (2003). Effects of attentional load on auditory scene analysis. Journal of Cognitive Neuroscience, 15, 1063-1073.

Anderson, C. M. B., \& Craik, F. I. M. (1974). The effect of a concurrent task on recall from primary memory. Journal of Verbal Learning and Verbal Behavior, 13, 107-113.

Ardila, A. (2003). Language representation and working memory with bilinguals. Journal of Communication Disorders, 36, 233-240.

Ardila, A., Rosselli, M., Ostrosky-Solis, F., Marcos, J., Granda, G., \& Soto, M. (2000). Syntactic comprehension verbal memory and calculation abilities in Spanish-English bilinguals. Applied Neuropsychology, 7, 3-16.

Bilger, R. B., Nuetzel, M. J., Rabinowitz, W. M., \& Rzeczkowski, C. (1984). Standardization of a test of speech perception in noise. Journal of Speech and Hearing Research, 27, 32-48.

Bonnel, A., \& Hafter, E. R. (1998). Divided attention between simultaneous auditory and visual signals. Perception and Psychophysics, 60, 179-190.

Bregman, A. S. (1990). Auditory scene analysis: The perceptual organization of sound. Cambridge, MA: MIT Press.

Brungart, D. S. (2001). Informational and energetic masking effects in the perception of two simultaneous talkers. Journal of the Acoustical Society of
America, 109, 1101-1109.

Carlyon, R. P., Cusack, R., Foxton, J. M., \& Robertson, I. H. (2001). Effects of attention and unilateral neglect on auditory stream segregation. Journal of Experimental Psychology: Human Perception and Performance, 27, 115-127.

Carlyon, R. P., Plack, C. J., Fantini, D. A., \& Cusack, R. (2003). Cross-modal and non-sensory influences on auditory streaming. Perception, 32, 1393-1402.

Clark, J. G. (1981). Uses and abuses of hearing loss classification. American Speech-Language-Hearing Association, 23, 493-500.

Craik, F. I. M., \& Byrd, M. (1982). Aging and cognitive deficits: The role of attentional resources. In F. I. M. Craik \& S. Trehub (Eds.), Aging and cognitive processes (pp. 191-211). Hillsdale, NJ: Lawrence Erlbaum Associates.

Craik, F. I. M., Govoni, R., Naveh-Benjamin, M., \& Anderson, N. D. (1996). The effects of divided attention on encoding and retrieval processes in human memory. Journal of Experimental Psychology: General, 125, 159-180.

Durlach, N. I., Mason, C. R., Shinn-Cunningham, B. G., Arbogast, T. L., Colburn, H. S., \& Kidd, G., Jr. (2003). Informational masking: Counteracting the effects of stimulus uncertainty by decreasing target-masker similarity. Journal of the Acoustical Society of America, 114, 368-379.

Freyman, R. L., Balakrishnan, U., \& Helfer, K. S. (2001). Spatial release from informational masking in speech recognition. Journal of the Acoustical Society of America, 109, 2112-2122.

Hasher, L., \& Zacks, R. T. (1979). Automatic and effortful processes in memory. Journal of Experimental Psychology: General, 108, 356-388.

Hasher, L., Zacks, R. T., \& May, C. P. (1999). Inhibitory control, circadian arousal, and age. In D. Gopher \& A. Koriat (Eds.), Attention and performance: XVII. Cognitive regulation of performance: Interaction of theory and application (pp. 653-675). Cambridge, MA: The MIT Press.

Kucera, H., \& Francis, W. N. (1967). Computational analysis of present-day American English. Providence, RI: Brown University Press.

Levitt, H. (1971). Transformed up-down methods in psychoacoustics. Journal of the Acoustical Society of America, 49, 467-477.

Li, L., Daneman, M., Qi, J. G., \& Schneider, B. A. (2004). Does the information content of an irrelevant source differentially affect spoken word recognition in younger and older adults? Journal of 
Experimental Psychology: Human Perception and Performance, 30, 1077-1091.

Luce, P. A., Feustel, T. C., \& Pisoni, D. B. (1983). Capacity demands in short-term memory for synthetic and natural speech. Human Factors, 25, $17-32$.

Madigan, S. A., \& McCabe, L. (1971). Perfect recall and total forgetting: A problem for models of short-term memory. Journal of Verbal Learning and Verbal Behavior, 10, 101-106.

Mangels, J. A., Craik, F. I. M., Levine, B., Schwartz, M. L., \& Stuss, D. T. (2002). Effects of divided attention on episodic memory in chronic traumatic brain injury: A function of severity and strategy. Neuropsychologia, 40, 2369-2385.

McCoy, S. L., Tun, P. A., Cox, L. C., Colangelo, M., Stewart, R. A., \& Wingfield, A. (2005). Hearing loss and perceptual effort: Downstream effects on older adults' memory for speech. The Quarterly Journal of Experimental Psychology, 58A, 22-33.

McFadden, D., \& Wright, B. A. (1990). Temporal decline of masking and comodulation detection differences. Journal of the Acoustical Society of America, 88, 711-724.

Murphy, D. R., Craik, F. I. M., Li, K. Z. H., \& Schneider, B. A. (2000). Comparing the effects of aging and background noise on short-term memory performance. Psychology and Aging, 15, 323-334.

Murphy, D. R., Daneman, M., \& Schneider, B. A. (2006). Why do older adults have difficulty following conversations? Psychology and Aging, 21, 49-61.

Naveh-Benjamin, M. (2001). The effects of divided attention on encoding processes: Underlying mechanisms. In M. Naveh-Benjamin, M. Moscovitch, \& H. L. Roediger, III (Eds.), Perspectives on human memory and cognitive aging: Essays in honour of Fergus Craik (pp. 193-207). New York: Psychology Press.

Peelle, J. E., \& Wingfield, A. (2005). Dissociations in perceptual learning revealed by adult age differences in adaptation to time-compressed speech. Journal of Experimental Psychology: Human Perception and Performance, 31, 1315-1330.
Pichora-Fuller, M. K., Schneider, B. A., \& Daneman, M. (1995). How young and old adults listen to and remember speech in noise. Journal of the Acoustical Society of America, 97, 593-608.

Rabbitt, P. M. A. (1968). Channel-capacity, intelligibility and immediate memory. Quarterly Journal of Experimental Psychology, 20, 241-248.

Rabbitt, P. M. A. (1991). Mild hearing-loss can cause apparent memory failures which increase with age and reduce with IQ. Acta Oto-Laryngologica (Suppl. 476), 167-176.

Rabinowitz, J. C., Craik, F. I. M., \& Ackerman, B. P. (1982). A processing resource account of age differences in recall. Canadian Journal of Psychology, 36, 325-344.

Schneider, B. A., Daneman, M., Murphy, D. R., \& Kwong See, S. (2000). Listening to discourse in distracting settings: The effects of aging. Psychology and Aging, 15, 110-125.

Schneider, B. A., Li, L., \& Daneman, M. (in press). How competing speech interferes with speech comprehension in everyday listening situations. Journal of the American Academy of Audiology.

Sussman, E., Winkler, I., Huotilainen, M., Ritter, W., \& Näätänen, R. (2002). Top-down effects can modify the initially stimulus-driven auditory organization. Cognitive Brain Research, 13, 393-405.

Troyer, A. K., \& Craik, F. I. M. (2000). The effect of divided attention on memory for items and their context. Canadian Journal of Experimental Psychology, 54, 161-171.

Wagener, K. C., \& Brand, T. (2005). Sentence intelligibility in noise for listeners with normal hearing and hearing impairment: Influence of measurement procedure and masking parameters. International Journal of Audiology, 44, 144-156.

Wright, B. A. (1997). Detectability of simultaneously masked signals as a function of masker bandwidth and configuration for different delays. Journal of the Acoustical Society of America, 101, 420-429.

Zwicker, E. (1965). Temporal effects in simultaneous masking by white-noise bursts. Journal of the Acoustical Society of America, 37, 653-663. 